\title{
Dynamical response to a stationary tidal field
}

\author{
Philippe Landry and Eric Poisson \\ Department of Physics, University of Guelph, Guelph, Ontario, N1G 2W1, Canada
}

(Dated: October 30, 2015)

\begin{abstract}
We demonstrate that a slowly rotating compact body subjected to a stationary tidal field undergoes a dynamical response, in which the fluid variables and the interior metric vary on the time scale of the rotation period. This dynamical response requires the tidal field to have a gravitomagnetic component generated by external mass currents; the response to a gravitoelectric tidal field is stationary. We confirm that in a calculation carried out to first order in the body's rotation, the exterior geometry bears no trace of this internal dynamics; it remains stationary in spite of the time-dependent interior.
\end{abstract}

\section{INTRODUCTION AND SUMMARY}

A recent observation by Flanagan and Hinderer [1, 2], that the tidal deformation of neutron stars in inspiraling binaries could leave a measurable imprint on the gravitational waves they emit, has triggered a surge of activity to better understand the tidal deformation of compact bodies in general relativity. This led to a precise formulation of relativistic Love numbers [3 5], a computation of these Love numbers for realistic models of neutron stars and a thorough investigation of whether they can truly be measured in gravitational waves [6-19], the discovery of the remarkable $I$-Love- $Q$ relations [20-26], and the computation of tidal invariants to be inserted within point-particle actions to model the tidal response of an extended body [27-31].

To further contribute to this effort, we initiated a program to calculate the tidal deformation of slowly rotating bodies in general relativity. We began in Ref. 32] (Paper 0) with the specific case of a black hole, and continued in Ref. 33] (Paper I) with the determination of the exterior geometry of a material body; this objective was simultaneously pursued by Pani et al. in Ref. [34]. In this paper we examine the interior geometry of the material body, and determine the state of the fluid making up the body. This objective was also pursued by Pani et al. [35], but these authors reach dramatically different conclusions.

Our main result is delightfully surprising. We find that a slowly rotating body subjected to a stationary tidal field undergoes a dynamical response, in which the fluid variables and the interior metric vary with time; the response occurs on the time scale of the rotation period. We also confirm that the exterior geometry of the slowly rotating body bears no trace of this internal dynamics - it is perfectly stationary.

These effects were not seen in Ref. [35]. There are two reasons for this discrepancy. The first and most important is that these authors keep the fluid artificially static by demanding that the velocity perturbation created by the tidal field vanishes. We require instead the perturbation of the vorticity tensor to vanish, which is accomplished by a nonzero velocity field. We argue below (and more fully in Sec. III) that our adopted state for the fluid is the one naturally established when the body is assumed to begin in an unperturbed state. A second reason for the discrepancy is that these authors consider tidal environments that are symmetric about the body's rotation axis. As we shall see below (in Sec. IVB), the time dependence would be revealed in non-axisymmetric situations, even under the static-fluid assumption. And indeed, consideration of non-axisymmetric tidal fields reveals that the static state is unphysical 1

To better explain the meaning of our results, we consider (for now) the specific tidal environment created by a companion body of mass $M^{\prime}$ placed on a circular orbit of radius $b$, velocity $v$, and angular velocity $\Omega_{\text {orb }}$ around our reference body, which has a mass $M$, radius $R$, and rotational angular velocity vector $\Omega^{a}$. To leading order in the tidal interaction and in a post-Newtonian expansion of the orbital motion, the tidal environment is described by the quadrupole moments

$$
\mathcal{E}_{a b}=-\frac{3 M^{\prime}}{b^{3}}\left(N_{a} N_{b}-\frac{1}{3} \delta_{a b}\right), \quad \mathcal{B}_{a b}=-\frac{3 M^{\prime} v}{b^{3}}\left(L_{a} N_{b}+N_{a} L_{b}\right),
$$

in which $N_{a}:=\left[\cos \left(\Omega_{\mathrm{orb}} t\right), \sin \left(\Omega_{\mathrm{orb}} t\right), 0\right]$ points from the reference body to the companion, while $L_{a}:=[0,0,1]$ is normal to the orbital plane. The tensor $\mathcal{E}_{a b}$ is known as the gravitoelectric tidal moment, and to leading order in the tidal interaction, it provides a complete description of the tidal environment in Newtonian gravity. The tensor $\mathcal{B}_{a b}$ is

\footnotetext{
${ }^{1}$ It is known that a non-axisymmetric tidal field exerts a torque that decreases the body's angular momentum over a very long time scale determined in part by the source of dissipation within the body. This is not the effect discussed here, which occurs over the short time scale of the rotation period.
} 
known as the gravitomagnetic tidal moment, and it has no analogue in the Newtonian theory. The phase of the tidal moments varies on the time scale of the orbital period, and the amplitude varies on a much longer radiation-reaction time scale. In this work we assume that the orbital period is much longer than the body's rotational period. In this regime the body's response to the tidal field occurs over the short time scale, and the variation of the tidal environment over the long time scale can be neglected. This is the stationary regime considered in this work. For our purposes we therefore take $\mathcal{E}_{a b}$ and $\mathcal{B}_{a b}$ to be time-independent, but we do not assume that they take the specific forms displayed in Eq. (1.1); except for the stationarity assumption, our tidal environment is generic.

We create the tidal environment by immersing the body in a spacetime that is not asymptotically flat, but modified to account for the presence of $\mathcal{E}_{a b}$ and $\mathcal{B}_{a b}$. The details of the construction are provided in Paper I [33], and our objective in this paper is to extend the exterior solution of Paper I to the body's interior. This requires solving the Einstein field equations for the metric, together with the relativistic Euler equation for the fluid variables. This, in turn, requires the specification of the fluid's state. For this purpose we rely on the fact that the amplitude of the tidal moments $\mathcal{E}_{a b}$ and $\mathcal{B}_{a b}$ reflects an orbital evolution over a very long radiation-reaction time scale. The reference body can therefore be taken to be isolated in the remote past, and to have begun in an unperturbed state in the absence of an initial tidal field. This assumption is sufficient to specify the state of the fluid at any later time: The Lagrangian perturbation $\Delta \omega_{\alpha \beta}$ of the vorticity tensor vanishes in the initial state, and conservation of vorticity guarantees that $\Delta \omega_{\alpha \beta}$ continues to vanish at later times. This observation is a key physics input, and as we shall see in Sec. III, conservation of vorticity implies that fluid motions develop as the tidal field gets established over the radiation-reaction time scale.

Solving the field and fluid equations reveals that a subset of metric and fluid variables must depend on time, in spite of the fact that the external tidal field is taken to be stationary. An example is

$$
g_{t r}=t k_{t r 1}^{\mathrm{d}}(r) \Omega^{a} \mathcal{B}_{a b} n^{b}+t k_{t r 1}^{\circ}(r) \Omega_{\langle a} \mathcal{B}_{b c\rangle} n^{a} n^{b} n^{c},
$$

where $k_{t r 1}^{\mathrm{d}}(r)$ and $k_{t r 1}^{\circ}(r)$ are functions of the radial coordinate $r$ that will be determined below, $n^{a}:=x^{a} / r$ is a unit vector that points away from the body's center-of-mass, and the angular brackets instruct to symmetrize all indices and remove all traces. The first term in Eq. (1.2) is a dipole $(\ell=1)$ perturbation, while the second term is an octupole $(\ell=3)$ perturbation; these result from the coupling between the dipole, rotational perturbation generated by $\Omega^{a}$ and the quadrupole, tidal perturbation generated by $\mathcal{B}_{a b}$. We find that all time-dependent terms in the metric and fluid variables require the existence of a gravitomagnetic field $\mathcal{B}_{a b}$. The gravitoelectric field $\mathcal{E}_{a b}$ does not provoke a dynamical response; all associated metric and fluid variables are time-independent. The time dependence displayed in Eq. (1.2) describes a steady growth. We point out, however, that our calculation is carried out to first order in $\Omega:=\left|\Omega^{a}\right|$, and that it cannot distinguish between $\Omega t$ and $\sin \Omega t$. We consider it likely that the time dependence is actually bounded.

The construction of Paper I guarantees that the exterior metric is stationary. The time-dependent metric functions are therefore required to be supported inside the body only, and to vanish outside the body. We show below (in Sec. IV] that this is indeed dictated by the fluid equations: the condition $\Delta p=0$ at $r=R$, where $\Delta p$ is the Lagrangian perturbation of the pressure, implies that $k_{t r 1}^{\mathrm{d}}(r)$ and $k_{t r 1}^{\circ}(r)$ must smoothly vanish at $r=R$. We point out that the stationary exterior is the consequence of a calculation carried out to first order in $\Omega$; a calculation taken to higher order would reveal the gravitational waves produced by the time-dependent interior.

The dynamical response of a slowly rotating body to a stationary tidal field is a startling outcome. Before proceeding with a detailed demonstration of this result, we attempt an intuitive explanation. The following argument is not meant to be rigorous or precise, but we believe that it does capture the essence of the phenomenon.

The dynamics of a perturbed fluid can be described in terms of a Lagrangian displacement $\boldsymbol{\xi}$, which maps the position of a perturbed fluid element to its original, unperturbed position. The displacement field satisfies a complicated differential equation, and this equation can be integrated by decomposing $\boldsymbol{\xi}$ in a complete basis of normal modes $\boldsymbol{z}_{\lambda}$, with $\lambda$ denoting a mode label. Writing $\boldsymbol{\xi}(t, \boldsymbol{x})=\sum_{\lambda} a_{\lambda}(t) \boldsymbol{z}_{\lambda}(\boldsymbol{x})$ and inserting within the fluid equation produces a mode equation of the form

$$
\ddot{a}_{\lambda}+\omega_{\lambda}^{2} a_{\lambda}=f_{\lambda},
$$

where an overdot indicates differentiation with respect to $t, \omega_{\lambda}$ is the mode's natural frequency, and $f_{\lambda}=\int \boldsymbol{F} \cdot \boldsymbol{z}_{\lambda} d^{3} x$ is the forcing function, obtained by evaluating an overlap integral between the external perturbation $\boldsymbol{F}$ and each mode function. The mode equation, of course, is the familiar equation that governs a simple harmonic oscillator driven by an external force $f_{\lambda}$.

In our context $f_{\lambda}$ is time-independent, and the expected stationary solution to the mode equation is obtained by balancing the external force with the oscillator's restoring force; we get $a_{\lambda}=\omega_{\lambda}^{-2} f_{\lambda}$. But what if the mode spectrum includes zero-frequency modes? In this case there is no restoring force to balance out the external force, and the solution can no longer be stationary. Choosing an unperturbed state at $t=0$, we have instead $a_{\lambda}=\frac{1}{2} f_{\lambda} t^{2}$. The 
displacement field grows quadratically with time, and it gives rise to a velocity field proportional to $t$. This is entirely analogous to our situation; the velocity field found in Secs. [II] and [V] does indeed grow linearly with time, just as $g_{t r}$ in Eq. (1.2).

Whence cometh the zero-frequency modes? The existence of such modes in relativistic fluids is well documented (see Ref. [36] for a clear presentation), and we summarize the situation more fully in Sec. IVA] For the polar (even-parity) perturbations of a static, spherical body, there exists an infinite class of zero-frequency $g$-modes describing a steady velocity field within the body. For the axial (odd-parity) perturbations, there exists an infinite class of zero-frequency $r$-modes, which also describe a steady velocity field. We believe that these modes are implicated in the tidal response of a slowly rotating body. In the absence of rotation, the overlap integral between the external tidal field and each zero-mode function vanishes, and the response is stationary. In the presence of rotation, however, the forcing function no longer vanishes, and the tidal response is dynamical. Our detailed computations indicate that the overlap integral continues to vanish when $\mathcal{B}_{a b}=0$; the dynamical response requires a gravitomagnetic tidal field.

It seems plausible that the time-dependent metric and fluid variables discussed in this work can be related to the rotational modes of relativistic stars described by Lockitch, Andersson, and Friedman [36, 37]; these generalize the famous $r$-modes of Newtonian gravity. We shall not attempt a precise identification, but the thought evokes a tantalizing possibility. Like the $r$-modes [38], the rotational modes are susceptible to the Chandrasekhar-SchutzFriedman instability [39, 40], and this suggests that an external tidal field can naturally create rotational modes that will be driven unstable by the emission of gravitational radiation. This could be a significant factor in the tidal interaction of neutron stars. We shall not, however, speculate any further.

Our main objective with this paper is to demonstrate the existence of the dynamical response of a slowly rotating body to a stationary tidal field. Because the dynamical response is associated exclusively with the gravitomagnetic field $\mathcal{B}_{a b}$, we shall ignore the gravitoelectric field $\mathcal{E}_{a b}$ altogether. We begin our technical developments in Sec. II], where we introduce the background metric of a static, spherically symmetric body, set up the rotational and tidal perturbations, and describe the coupling between these perturbations. In Sec. III we introduce the fluid variables, and work out the consequences of the key fluid equation, $\Delta \omega_{\alpha \beta}=0$. In Sec. IV we manipulate the Einstein field equations to deliver a complete set of equations for all metric and fluid variables. We leave the task of actually integrating these equations to a forthcoming publication 2

\section{SPACETIME OF A TIDALLY DEFORMED, SLOWLY ROTATING BODY}

The metric of a tidally deformed, slowly rotating body is constructed as a perturbation of the background metric of an undeformed and nonrotating body. It is assumed that the unperturbed body is in hydrostatic equilibrium, and the background metric is expressed in the standard form

$$
d s^{2}=-e^{2 \psi} d t^{2}+f^{-1} d r^{2}+r^{2} d \Omega^{2},
$$

where $\psi=\psi(r), f=1-2 m(r) / r$, and $d \Omega^{2}:=d \theta^{2}+\sin ^{2} \theta d \phi^{2}$. The Einstein field equations imply that $\psi$ and $m$ are solutions to

$$
\frac{d m}{d r}=4 \pi r^{2} \mu, \quad \frac{d \psi}{d r}=\frac{m+4 \pi r^{3} p}{r^{2} f},
$$

where $\mu$ is the fluid's energy density (the sum of mass density $\rho$ and density of internal energy $\epsilon$ ), and $p$ is the pressure. The condition of hydrostatic equilibrium is

$$
\frac{d p}{d r}=-\frac{(\mu+p)\left(m+4 \pi r^{3} p\right)}{r^{2} f} .
$$

These equations must be supplemented with an equation of state, which we take to be of the form $p=p(\rho)$ and $\epsilon=\epsilon(\rho)$; the fluid is thus assumed to have a constant specific entropy. The interior metric must be matched to the Schwarzschild exterior metric at $r=R$, at which $p=0$.

The slow rotation of the body is incorporated as a linear perturbation of the background metric. We assume that the rotation is rigid, and the metric perturbation $p_{\alpha \beta}$ has the single nonvanishing component 3

$$
p_{t \phi}^{\text {rotation }}=-\Omega(1-\omega) r^{2} \sin ^{2} \theta,
$$

\footnotetext{
${ }^{2}$ P. Landry and E. Poisson, in preparation.

${ }^{3}$ Our function $\Omega \omega(r)$ is typically denoted $\bar{\omega}(r)$ in the literature.
} 
where $\Omega$ is the body's angular velocity, and $\omega(r)$ is a solution to

$$
r^{2} f \frac{d^{2} \omega}{d r^{2}}+\left[4 f-4 \pi r^{2}(\mu+p)\right] r \frac{d \omega}{d r}-16 \pi r^{2}(\mu+p) \omega=0 .
$$

The interior solution is matched at $r=R$ to the exterior solution $\omega_{\text {ext }}=1-2 I / r^{3}$, where $I:=J / \Omega$ is the body's moment of inertia.

As was explained in Sec. [I. we place the body in a tidal environment characterized by a gravitomagnetic quadrupole moment $\mathcal{B}_{a b}$, a Cartesian symmetric-tracefree (STF) tensor that we take to be independent of time - the tidal environment is stationary. The five independent components of $\mathcal{B}_{a b}$ can be packaged in spherical-harmonic coefficients $\mathcal{B}_{\mathrm{m}}^{\mathrm{q}}$, with the label $\mathrm{m}$ ranging over the five values associated with spherical harmonics of degree $\ell=2$. The precise packaging is described in Table I of Paper I [33].

The tidal perturbation is conveniently decomposed in spherical harmonics. Because the perturbation created by $\mathcal{B}_{a b}$ is axial (odd-parity) in nature, the decomposition is accomplished with the odd-parity vectorial harmonics

$$
X_{A}^{\ell \mathrm{m}}:=-\epsilon_{A}^{B} D_{B} Y^{\ell \mathrm{m}} .
$$

Here, uppercase Latin indices $A, B, C, \cdots$ range over the angular variables $\theta$ and $\phi, Y^{\ell m}$ are the usual sphericalharmonic functions (with the normalization adopted in Table II of Paper I), $\Omega_{A B}=\operatorname{diag}\left(1, \sin ^{2} \theta\right)$ is the metric on a unit two-sphere, $D_{A}$ is the covariant-derivative operator compatible with this metric, and $\epsilon_{A B}$ is the Levi-Civita tensor on the unit two-sphere $\left(\epsilon_{\theta \phi}=\sin \theta\right)$; it is our convention that an uppercase Latin index is raised with $\Omega^{A B}$, the matrix inverse to $\Omega_{A B}$.

We adopt the Regge-Wheeler gauge, and find (see Ref. 41] for a derivation) that the only nonvanishing component of the tidal perturbation is given by

$$
p_{t A}^{\mathrm{tidal}}=b_{t}^{\mathrm{q}}(r) \mathcal{B}_{A}^{\mathrm{q}}
$$

where

$$
\mathcal{B}_{A}^{\mathrm{q}}:=\frac{1}{2} \sum_{\mathrm{m}} \mathcal{B}_{\mathrm{m}}^{\mathrm{q}} X_{A}^{2 \mathrm{~m}}
$$

The Einstein field equations imply that the radial function $b_{t}^{\mathrm{q}}$ satisfies

$$
r^{2} f \frac{d^{2} b_{t}^{\mathrm{q}}}{d r^{2}}-4 \pi r^{3}(\mu+p) \frac{d b_{t}^{\mathrm{q}}}{d r}-2\left[3-2 m / r \mp 4 \pi r^{2}(\mu+p)\right] b_{t}^{\mathrm{q}}=0 .
$$

The choice of sign in this equation depends on the assumed state of the fluid; the upper sign corresponds to an irrotational fluid with internal motions, while the lower sign corresponds to a static fluid with no internal motions. As we shall explain below, the upper sign is the appropriate choice for our purposes in this work.

We next allow the (dipole) rotational perturbation of Eq. (2.4) and the (quadrupole) tidal perturbation of Eq. (2.7) to source a second-order perturbation. We continue to work to first order in $\Omega$ and to first order in $\mathcal{B}_{a b}$, but we introduce terms of order $\Omega \mathcal{B}_{a b}$ in the perturbed metric. The composition of the $\ell=1$ and $\ell=2$ spherical harmonics is reflected in the bilinear moment:5

$$
\mathcal{K}_{a}:=\mathcal{B}_{a b} \Omega^{b}, \quad \hat{\mathcal{B}}_{a b}:=2 \Omega^{c} \epsilon_{c d(a} \mathcal{B}^{d}{ }_{b)}, \quad \mathcal{K}_{a b c}:=\mathcal{B}_{\langle a b} \Omega_{c\rangle},
$$

where $\Omega_{a}:=[0,0, \Omega]$ is the angular-velocity vector, $\epsilon_{a b c}$ is the antisymmetric permutation symbol, the angular brackets indicate the STF operation (symmetrize all indices and remove all traces), and all lowercase Latin indices are raised and lowered with the Euclidean metric. The independent components of the STF tensors $\mathcal{K}_{a}, \hat{\mathcal{B}}_{a b}$, and $\mathcal{K}_{a b c}$ can also be packaged in spherical-harmonic coefficients $\mathcal{K}_{\mathrm{m}}^{\mathrm{d}}, \hat{\mathcal{B}}_{\mathrm{m}}^{\mathrm{q}}$, and $\mathcal{K}_{\mathrm{m}}^{\mathrm{o}}$, respectively; the precise definitions are displayed in Table I of Paper I (with the change of notation described in footnote 5).

We continue to adopt the Regge-Wheeler gauge for the second-order perturbation, and for the $\ell=1$ terms generated by $\mathcal{K}_{a}$ we adopt the extension formulated by Campolattaro and Thorne [42]. We observe that $\mathcal{K}_{a}$ and $\mathcal{K}_{a b c}$ create

\footnotetext{
${ }^{4}$ Our notation for $b_{t}^{\mathrm{q}}(r)$ differs from the one adopted in Sec. IV of Paper I; we have that $b_{t}^{\mathrm{q}}[$ here $]=\frac{2}{3} r^{3} b_{t}^{\mathrm{q}}[$ Paper I]

${ }^{5}$ We use the same notation as in Paper I for the bilinear moments, but construct them with $\Omega^{a}$ instead of $\chi^{a}$, the dimensionless angular-momentum vector. The moments therefore differ by a factor $\chi / \Omega=I / M^{2}$.
} 
polar (even-parity) perturbations, while $\hat{\mathcal{B}}_{a b}$ creates axial (odd-parity) perturbations, and express the perturbation a: 6

$$
\begin{aligned}
& p_{t t}^{\text {bilinear }}=k_{t t}^{\mathrm{d}}(t, r) \mathcal{K}^{\mathrm{d}}+k_{t t}^{\circ}(t, r) \mathcal{K}^{\circ}, \\
& p_{t r}^{\text {bilinear }}=k_{t r}^{\mathrm{d}}(t, r) \mathcal{K}^{\mathrm{d}}+k_{t r}^{\circ}(t, r) \mathcal{K}^{\circ}, \\
& p_{r r}^{\text {bilinear }}=k_{r r}^{\mathrm{d}}(t, r) \mathcal{K}^{\mathrm{d}}+k_{r r}^{\circ}(t, r) \mathcal{K}^{\circ}, \\
& p_{t A}^{\text {bilinear }}=\hat{b}_{t}^{\mathrm{q}}(t, r) \hat{\mathcal{B}}_{A}^{\mathrm{q}}, \\
& p_{r A}^{\text {bilinear }}=\hat{b}_{r}^{\mathrm{q}}(t, r) \hat{\mathcal{B}}_{A}^{\mathrm{q}}, \\
& p_{A B}^{\text {bilinear }}=k^{\circ}(t, r) \Omega_{A B} \mathcal{K}^{\circ},
\end{aligned}
$$

where

$$
\mathcal{K}^{\mathrm{d}}=\sum_{\mathrm{m}} \mathcal{K}_{\mathrm{m}}^{\mathrm{d}} Y^{1 \mathrm{~m}}, \quad \mathcal{K}^{\circ}=\sum_{\mathrm{m}} \mathcal{K}_{\mathrm{m}}^{\mathrm{o}} Y^{3 \mathrm{~m}}, \quad \hat{\mathcal{B}}_{A}^{\mathrm{q}}=\frac{1}{2} \sum_{\mathrm{m}} \hat{\mathcal{B}}_{\mathrm{m}}^{\mathrm{q}} X_{A}^{2 \mathrm{~m}}=-\Omega \partial_{\phi} \mathcal{B}_{A}^{\mathrm{q}}
$$

It should be noticed that we allow $\left\{k_{t t}^{\mathrm{d}}, \cdots, k^{\circ}\right\}$ to be functions of both $t$ and $r$, and that $\hat{\mathcal{B}}_{A}^{\mathrm{q}}$ vanishes when the tidal environment is axisymmetric.

\section{PERTURBED FLUID}

The perturbations created by $\mathcal{B}_{a b}, \mathcal{K}_{a}, \hat{B}_{a b}$, and $\mathcal{K}_{a b c}$ disturb the fluid, and we give this disturbance a Lagrangian formulation as summarized, for example, in Sec. 2.2 of Ref. [43]. We assume that the fluid is barotropic, which means that the equation of state of the perturbed fluid is the same as for the unperturbed fluid.

The Lagrangian displacement vector $\xi_{\alpha}$ is decomposed as

$$
\begin{aligned}
\xi_{r} & =\xi_{r}^{\mathrm{d}}(t, r) \mathcal{K}^{\mathrm{d}}+\xi_{r}^{\circ}(t, r) \mathcal{K}^{\circ}, \\
\xi_{A} & =\xi^{\mathrm{q}}(t, r) \mathcal{B}_{A}^{\mathrm{q}}+\xi^{\mathrm{d}}(t, r) \mathcal{K}_{A}^{\mathrm{d}}+\hat{\xi}^{\mathrm{q}}(t, r) \hat{\mathcal{B}}_{A}^{\mathrm{q}}+\xi^{\circ}(t, r) \mathcal{K}_{A}^{\circ},
\end{aligned}
$$

and the Eulerian perturbation of the velocity vector is decomposed as

$$
\begin{aligned}
\delta u_{r} & =v_{r}^{\mathrm{d}}(t, r) \mathcal{K}^{\mathrm{d}}+v_{r}^{\circ}(t, r) \mathcal{K}^{\circ}, \\
\delta u_{A} & =v^{\mathrm{q}}(r) \mathcal{B}_{A}^{\mathrm{q}}+v^{\mathrm{d}}(t, r) \mathcal{K}_{A}^{\mathrm{d}}+\hat{v}^{\mathrm{q}}(t, r) \hat{\mathcal{B}}_{A}^{\mathrm{q}}+v^{\circ}(t, r) \mathcal{K}_{A}^{\circ} ;
\end{aligned}
$$

the time component of $\xi_{\alpha}$ plays no role in our discussion, and $\delta u_{t}$ can be related to the other components by properly normalizing the perturbed velocity vector. The various expansion coefficients are related by

$$
\begin{aligned}
& v^{\mathrm{q}}=e^{-\psi}\left(\partial_{t} \xi^{\mathrm{q}}+b_{t}^{\mathrm{q}}\right), \\
& v_{r}^{\mathrm{d}}=e^{-\psi}\left(\partial_{t} \xi_{r}^{\mathrm{d}}+k_{t r}^{\mathrm{d}}\right), \\
& v^{\mathrm{d}}=e^{-\psi} \partial_{t} \xi^{d}, \\
& \hat{v}^{\mathrm{q}}=e^{-\psi}\left(\partial_{t} \hat{\xi}^{\mathrm{q}}-\xi^{\mathrm{q}}+\hat{b}_{t}^{\mathrm{q}}\right), \\
& v_{r}^{\mathrm{o}}=e^{-\psi}\left(\partial_{t} \xi_{r}^{\mathrm{o}}+k_{t r}^{\circ}\right), \\
& v^{\circ}=e^{-\psi} \partial_{t} \xi^{\circ} .
\end{aligned}
$$

The Eulerian perturbation of the pressure is expressed as

$$
\delta p=p^{\mathrm{d}}(t, r) \mathcal{K}^{\mathrm{d}}+p^{\circ}(t, r) \mathcal{K}^{\circ},
$$

and the barotropic assumption ensures that the perturbations in energy density $\mu$ and specific enthalpy $h$ are given by $\delta \mu=(d \mu / d p) \delta p$ and $\delta h=h(\mu+p)^{-1} \delta p$, respectively. We recall that the Lagrangian perturbation $\Delta Q$ of a fluid

\footnotetext{
${ }^{6}$ In addition to the change of notation described previously for the bilinear moments, we note that our notation for the various functions differs from the one adopted in Paper I. We have $k_{t t}^{\mathrm{d}}\left[\right.$ here] $=r^{2} k_{t t}^{\mathrm{d}}$ [Paper I], $k_{r r}^{\mathrm{d}}[$ here $]=r^{2} k_{r r}^{\mathrm{d}}\left[\right.$ Paper I], $k_{t t}^{\circ}\left[\right.$ here] $=-r^{2} k_{t t}^{\circ}[$ Paper I], $k_{r r}^{\circ}[$ here $]=-r^{2} k_{r r}^{\circ}\left[\right.$ Paper I], $k^{\circ}[$ here $]=r^{4} k^{\circ}$ [Paper I], $\hat{b}_{t}^{\mathrm{q}}[$ here $]=-r^{3} \hat{b}_{t}^{\mathrm{q}}\left[\right.$ Paper I], and $\hat{b}_{r}^{\mathrm{q}}[$ here $]=-r^{3} \hat{b}_{r}^{\mathrm{q}}[$ Paper I].
} 
variable $Q$ is related to its Eulerian perturbation $\delta Q$ by $\Delta Q=\delta Q+\mathcal{L}_{\xi} Q$, where $\mathcal{L}_{\xi}$ indicates Lie differentiation in the direction of $\xi^{\alpha}$.

The state of the fluid is constrained by the fact that the vorticity tensor $\omega_{\alpha \beta}:=\nabla_{\alpha}\left(h u_{\beta}\right)-\nabla_{\beta}\left(h u_{\alpha}\right)$ is conserved along the fluid world lines: $\mathcal{L}_{u} \omega_{\alpha \beta}=0$; see, for example, Sec. 1.1 of Ref. [43] for a derivation. Taking a Lagrangian perturbation of this equation gives rise to $\mathcal{L}_{u} \Delta \omega_{\alpha \beta}=0$, the statement that $\Delta \omega_{\alpha \beta}$ is conserved along the fluid world lines. To implement this condition we imagine that in spite of our assumption of stationarity, the tidal perturbation was switched on adiabatically in the remote past, so that the fluid began in an unperturbed state 7 In this initial state $\Delta \omega_{\alpha \beta}=0$, and the conservation statement implies that $\Delta \omega_{\alpha \beta}$ continues to vanish on each world line. Conservation of vorticity therefore guarantees that

$$
\Delta \omega_{\alpha \beta}=0
$$

at any time throughout the fluid.

The conditions of Eq. (3.5) have far-reaching consequences. We note first that the angular components of this equation imply that

$$
v^{\mathrm{q}}=0 .
$$

This, we recall, is the piece of $\delta u_{A}$ associated with the tidal perturbation of a nonrotating body, and the vorticity constraint implies the existence of internal motions within the fluid [41, 44]. These motions are described by $\delta u^{A}=-\frac{1}{2} r^{-2} e^{-\psi} b_{t}^{q}(r) \Omega^{A B} \mathcal{B}_{B}^{q}$, and they are gradually established as the tidal field is adiabatically switched on. The assignment of Eq. (3.6) dictates the choice of upper sign in Eq. (2.9). From $v^{q}=0$ and Eq. (3.3a) we next find that $\xi^{\mathrm{q}}=-t b_{t}^{\mathrm{q}}(r)$.

The angular components of Eq. (3.5) also produce $\hat{v}^{\mathrm{q}}=-\frac{1}{3} e^{-\psi} \omega \xi^{\mathrm{q}}$, in which we insert our previous result for $\xi^{\mathrm{q}}$. We arrive at

$$
\hat{v}^{\mathrm{q}}=\frac{1}{3} t e^{-\psi} \omega(r) b_{t}^{\mathrm{q}}(r)
$$

the striking statement that the response of a slowly rotating body to a stationary tidal field is necessarily dynamical. The $r A$ components of Eq. (3.5) give

$$
\begin{aligned}
& v_{r}^{\mathrm{d}}=\frac{3}{5} t e^{-\psi}\left[\omega \frac{d b_{t}^{\mathrm{q}}}{d r}+2\left(\frac{d \omega}{d r}+\frac{r-4 m-8 \pi r^{3} p}{r^{2} f} \omega\right) b_{t}^{\mathrm{q}}\right]+\partial_{r} v^{\mathrm{d}}-\frac{m+4 \pi r^{3} p}{r^{2} f} v^{\mathrm{d}} \\
& v_{r}^{\circ}=\frac{1}{3} t e^{-\psi}\left[2 \omega \frac{d b_{t}^{\mathrm{q}}}{d r}-\left(\frac{d \omega}{d r}+\frac{6 r-14 m-8 \pi r^{3} p}{r^{2} f} \omega\right) b_{t}^{\mathrm{q}}\right]+\frac{1}{3} \partial_{r} v^{\circ}-\frac{m+4 \pi r^{3} p}{3 r^{2} f} v^{\circ}
\end{aligned}
$$

and these also reveal the time dependence of the velocity field. The $t A$ components of Eq. (3.5) relate the pressure perturbations to other variables; we have

$$
\begin{aligned}
& p^{\mathrm{d}}=e^{-\psi}(\mu+p)\left[\frac{1}{2} e^{-\psi} k_{t t}^{\mathrm{d}}-\frac{3}{5} e^{-\psi}(1+\omega) b_{t}^{\mathrm{q}}-\partial_{t} v^{\mathrm{d}}\right], \\
& p^{\mathrm{o}}=e^{-\psi}(\mu+p)\left[\frac{1}{2} e^{-\psi} k_{t t}^{\mathrm{o}}+\frac{1}{3} e^{-\psi}(3-2 \omega) b_{t}^{\mathrm{q}}-\frac{1}{3} \partial_{t} v^{\mathrm{o}}\right] .
\end{aligned}
$$

This exhausts the information disclosed by Eq. (3.5); the remaining $\operatorname{tr}$ component is redundant.

\section{FIELD EQUATIONS}

\section{A. Zero-frequency modes}

Before we proceed with an analysis of the Einstein field equations, we briefly recall the existence of two classes of zero-frequency modes for the perturbations of a static, spherically symmetric body. For the purposes of this discussion we set $\Omega=0$, switch off the external tidal field, and turn off the time-dependence of the perturbation variables. We summarize the presentation contained in Sec. III of Ref. [36].

\footnotetext{
${ }^{7}$ For our purposes in this discussion, the body's rotation is excluded from the perturbation and included in the unperturbed configuration; the perturbation refers exclusively to the tidal field.
} 
We first consider a polar (even-parity) perturbation described by the metric variables $\left\{k_{t t}, k_{t r}, k_{r r}, k\right\}$ and the fluid variables $\left\{v_{r}, v, p\right\}$; we suppress the multipole label (such as $\mathrm{d}$ and $\mathrm{o}$ ) to emphasize the fact that the discussion is not limited to the dipole and octupole perturbations examined in this work. The variables decouple into the groups $\left\{k_{t t}, k_{r r}, k, p\right\}$ and $\left\{k_{t r}, v_{r}, v\right\}$, and it can be shown that for a homogeneous perturbation (not driven by an external tidal field), the variables belonging to the first group vanish. When the fluid is barotropic, however, the variables belonging to the second group admit an infinity of solutions, each one characterized by a freely specifiable $v_{r}$. These define the class of zero-frequency $g$-modes, which were first identified by Thorne [45].

We next consider an axial (odd-parity) perturbation described by the metric and fluid variables $\left\{\hat{b}_{t}, \hat{b}_{r}, \hat{v}\right\}$, where we again suppress the multipole label (such as q). In this case the field equations reveal the existence of another infinity of solutions, each one characterized by a freely specifiable $\hat{v}$ and a vanishing $\hat{b}_{r}$. These define the class of zero-frequency $r$-modes, which are not limited to barotropic fluids.

\section{B. Field equations: $\ell=2$}

We substitute the metric of Sec. II and the fluid variables of Sec. III into the Einstein field equations

$$
G_{\alpha \beta}=8 \pi T_{\alpha \beta},
$$

expand to first order in $\Omega$ and $\mathcal{B}_{\mathrm{m}}^{\mathrm{q}}$, and decompose each component in (scalar, vector, and tensor) spherical harmonics. The field equations for each multipole order decouple, and in this subsection we examine the $\ell=2$ sector associated with the variables $\left\{\hat{b}_{t}^{\mathrm{q}}, \hat{b}_{r}^{\mathrm{q}}, \hat{v}^{\mathrm{q}}\right\}$.

The velocity perturbation is eliminated with Eq. (3.7), and inspired by this relation, we assume that the metric variables can be at most linear in $t$, so that $\hat{b}_{t}^{\mathrm{q}}=\hat{b}_{t 0}^{\mathrm{q}}(r)+t \hat{b}_{t 1}^{\mathrm{q}}(r)$ and $\hat{b}_{r}^{\mathrm{q}}=\hat{b}_{r 0}^{\mathrm{q}}(r)+t \hat{b}_{r 1}^{\mathrm{q}}(r)$. The $r A$ components of the field equations then imply that $\hat{b}_{r 1}^{\mathrm{q}}=0$, and the $t A$ components give rise to a homogeneous differential equation for $\hat{b}_{t 0}^{\mathrm{q}}$. The solution to this equation represents an $r$-mode, and to simplify our solution we choose to eliminate this degree of freedom by setting $\hat{b}_{t 0}^{\mathrm{q}}=0$; the $r$-mode can be restored at will.

We are left with

$$
\hat{b}_{t}^{\mathrm{q}}(t, r)=t \hat{b}_{t 1}^{\mathrm{q}}(r), \quad \hat{b}_{r}^{\mathrm{q}}(t, r)=\hat{b}_{r 0}^{\mathrm{q}}(r),
$$

and the $t A$ components of the field equations give rise to

$$
r^{2} f \frac{d^{2} \hat{b}_{t 1}^{\mathrm{q}}}{d r^{2}}-4 \pi r^{3}(\mu+p) \frac{d \hat{b}_{t 1}^{\mathrm{q}}}{d r}-\frac{2}{r}\left[3 r-2 m-4 \pi r^{3}(\mu+p)\right] \hat{b}_{t 1}^{\mathrm{q}}-\frac{16 \pi}{3} r^{2}(\mu+p) \omega b_{t}^{\mathrm{q}}=0
$$

a differential equation that determines $\hat{b}_{t 1}^{\mathrm{q}}(r)$. The $r A$ components return

$$
\hat{b}_{r 0}^{\mathrm{q}}=\frac{1}{4} e^{-2 \psi}\left[r^{2} \frac{d \hat{b}_{t 1}^{\mathrm{q}}}{d r}-2 r \hat{b}_{t 1}^{\mathrm{q}}-r^{2}(1-\omega) \frac{d b_{t}^{\mathrm{q}}}{d r}-\frac{1}{3} r\left(r \frac{d \omega}{d r}+6 \omega-6\right) b_{t}^{\mathrm{q}}\right],
$$

an algebraic equation for $\hat{b}_{r 0}^{\mathrm{q}}(r)$.

The interior metric variables must be matched with the exterior solutions at $r=R$. The exterior metric was constructed in Paper I [33], and we have re-examined this construction to ensure that we didn't incorrectly eliminate time-dependent terms. The conclusion of this exercise is that the metric of Paper I requires no change; the exterior metric must be stationary, in spite of the time-dependent interior. This implies that $\hat{b}_{t 1}^{\mathrm{q}}$ must satisfy the boundary conditions 8

$$
\hat{b}_{t 1}^{\mathrm{q}}=0=\frac{d \hat{b}_{t 1}^{\mathrm{q}}}{d r} \quad \text { at } r=R,
$$

and that $\hat{b}_{r 0}^{\mathrm{q}}$ must match at $r=R$ the value listed in Table IV of Paper I (after taking into account the change of notation documented in footnotes 5 and 6 ). In addition, these functions must satisfy regularity conditions at $r=0$.

\footnotetext{
${ }^{8}$ Equation (4.5) may seem to provide too many conditions. This is not so. We assume that the equation of state approaches a polytropic form near the surface, so that $p \propto \mu^{1+1 / n}$, with $n>0$. The structure equations then imply that as $r \rightarrow R, p$ and $\mu$ approach zero as $p \propto(1-r / R)^{n+1}$ and $\mu \propto(1-r / R)^{n}$. Inspection of Eq. (4.3) finally reveals that if $\hat{b}_{t 1}^{\mathrm{q}}$ is to vanish at $r=R$, it must do so as $(1-r / R)^{2+n}$.
} 
It is interesting to reconsider the interior solution when the fluid is taken to be static, in the sense that it is prevented to move, in contradiction with our assumption in Eq. (3.5). In this case we must set $v^{q}=e^{-\psi} b_{t}$ and $\hat{v}^{\mathrm{q}}=e^{-\psi} \hat{b}_{t}^{\mathrm{q}}$ to ensure that $\delta u^{A}=0$, and the conservation equations $\nabla_{\beta} T^{\alpha \beta}=0$ imply that $\hat{b}_{t}^{\mathrm{q}}$ must be of the form $\hat{b}_{t}^{\mathrm{q}}(t, r)=\hat{b}_{t 0}^{\mathrm{q}}(r)+t b_{t}^{\mathrm{q}}(r)$; it is significant that the time-dependent term involves the driving function $b_{t}^{\mathrm{q}}(r)$. The field equations further imply that $\hat{b}_{t 0}^{q}(r)$ satisfies a homogeneous differential equation; the solution represents another $r$ mode, which we again have the freedom to eliminate. The matching with the exterior solution creates a contradiction: The interior expression for $\hat{b}_{t}^{\mathrm{q}}$ is necessarily time-dependent and does not vanish at $r=R$, but the exterior expression is necessarily stationary. We conclude that a static fluid is an unphysical configuration when a slowly rotating body is deformed by a stationary tidal field. We note that the contradiction is lifted when the tidal field is axisymmetric; in this case $\hat{\mathcal{B}}_{A}^{\mathrm{q}}=0$ and the variables $\left\{\hat{b}_{t}^{\mathrm{q}}, \hat{b}_{r}^{\mathrm{q}}, \hat{v}^{\mathrm{q}}\right\}$ are not defined. It is for this reason that the contradiction was not noticed by the authors of Ref. [35], who restricted their attention to axisymmetric tidal fields 9

\section{Field equations: $\ell=1$}

In this subsection we examine the $\ell=1$ sector of the perturbation, associated with the variables $\left\{k_{t t}^{\mathrm{d}}, k_{r r}^{\mathrm{d}}, p^{\mathrm{d}}\right\}$ and $\left\{k_{t r}^{\mathrm{d}}, v_{r}^{\mathrm{d}}, v^{\mathrm{d}}\right\}$. The variables $v_{r}^{\mathrm{d}}$ and $p^{\mathrm{d}}$ are eliminated with Eqs. (3.8) and (3.9), respectively, and we find that the $t A$ and $r A$ components of the field equations allow us to further eliminate $v^{\mathrm{d}}$ and $k_{r r}^{\mathrm{d}}$. We are left with $k_{t t}^{\mathrm{d}}$ from the first group of variables, and $k_{t r}^{\mathrm{d}}$ from the second group. We next assume the explicit forms $k_{t t}^{\mathrm{d}}(t, r)=k_{t t 0}^{\mathrm{d}}(r)$ and $k_{t r}^{\mathrm{d}}(t, r)=k_{t r 0}^{\mathrm{d}}(r)+t k_{t r 1}^{\mathrm{d}}(r)$, which ensure that all variables from the first group are time-independent, while those from the second group are linear in time. Then the $t r$ component of the field equations implies that $k_{t r 0}^{\mathrm{d} 0}$ satisfies a homogeneous differential equation. The solution to this equation represents a $g$-mode, and we simplify our solution by setting $k_{t r 0}^{\mathrm{d}}=0$; the freedom to incorporate the $g$-mode can be restored at will.

We are left with

$$
k_{t t}^{\mathrm{d}}(t, r)=k_{t t 0}^{\mathrm{d}}(r), \quad k_{t r}^{\mathrm{d}}(t, r)=t k_{t r 1}^{\mathrm{d}}(r),
$$

and these functions are determined by the $t r$ and $r r$ components of the field equations, respectively. We have

$$
\begin{aligned}
0= & r^{2} f \frac{d^{2} k_{t r 1}^{\mathrm{d}}}{d r^{2}}+\left[3\left(m-4 \pi r^{3} \mu\right)+\left(m+4 \pi r^{3} p\right) \frac{d \mu}{d p}\right] \frac{d k_{t r 1}^{\mathrm{d}}}{d r} \\
& -\frac{2}{r^{2} f}\left\{\left[1-10 \pi r^{2}(\mu+p)+16 \pi^{2} r^{4} p^{2}\right] r^{2}+4 \pi r^{3}(5 \mu+7 p) m-3 m^{2}-\left(m+4 \pi r^{3} p\right)^{2} \frac{d \mu}{d p}\right\} k_{t r 1}^{\mathrm{d}} \\
& -\frac{48 \pi}{5} r^{2}(\mu+p) \omega \frac{d b_{t}^{\mathrm{q}}}{d r}-\frac{96 \pi}{5}(\mu+p)\left[r^{2} \frac{d \omega}{d r}+\frac{r-4 m-8 \pi r^{3} p}{f} \omega\right] b_{t}
\end{aligned}
$$

and

$$
\begin{aligned}
0= & r \frac{d k_{t t 0}^{\mathrm{d}}}{d r}+\frac{2\left[m-2 \pi r^{3}(\mu+p)\right]\left(r-m+4 \pi r^{3} p\right)}{r f\left(m+4 \pi r^{3} p\right)} k_{t t 0}^{\mathrm{d}}-\frac{r^{2}\left(r-m+4 \pi r^{3} p\right)}{2\left(m+4 \pi r^{3} p\right)} \frac{d k_{t r 1}^{\mathrm{d}}}{d r} \\
& +\frac{\left[1+2 \pi r^{2}\left(1+4 \pi r^{2} p\right)(\mu-p)\right] r^{2}-\left[5+2 \pi r^{2}(\mu+p)\right] r m+5 m^{2}}{f\left(m+4 \pi r^{3} p\right)} k_{t r 1}^{\mathrm{d}} \\
& -\frac{3 r}{10}\left[\frac{r\left(r-m+4 \pi r^{3} p\right)}{m+4 \pi r^{3} p} \frac{d \omega}{d r}-4 \omega+4\right] \frac{d b_{t}^{\mathrm{q}}}{d r} \\
& -\frac{3\left(r-m+4 \pi r^{3} p\right)}{5\left(m+4 \pi r^{3} p\right)}\left[r \frac{d \omega}{d r}-4 \frac{m+2 \pi r^{3}(\mu+p)}{r f} \omega+4 \frac{m-2 \pi r^{3}(\mu+p)}{r f}\right] b_{t}^{\mathrm{q}} .
\end{aligned}
$$

The remaining variables are given by

$$
\begin{aligned}
k_{r r}^{\mathrm{d}} & =e^{-2 \psi}\left[-\frac{r^{2}}{r-m+4 \pi r^{3} p} \frac{d k_{t t 0}^{\mathrm{d}}}{d r}+\frac{1}{f} k_{t t 0}^{\mathrm{d}}+\frac{r^{2}}{r-m+4 \pi r^{3} p} k_{t r 1}^{\mathrm{d}}+\frac{6(1-\omega) r^{2}}{5\left(r-m+4 \pi r^{3} p\right)} \frac{d b_{t}^{\mathrm{q}}}{d r}-\frac{6(1-\omega)}{5 f} b_{t}^{\mathrm{q}}\right], \\
p^{\mathrm{d}} & =e^{-2 \psi}\left[\frac{f}{16 \pi} \frac{d k_{t r 1}^{\mathrm{d}}}{d r}+\frac{m-2 \pi r^{3}(\mu-p)}{8 \pi r^{2}} k_{t r 1}^{\mathrm{d}}+\frac{1}{2}(\mu+p) k_{t t 0}^{\mathrm{d}}-\frac{3}{5}(\mu+p)(1+\omega) b_{t}^{\mathrm{q}}\right]
\end{aligned}
$$

\footnotetext{
${ }^{9}$ Recall footnote 1 The time dependence discussed here occurs over the short time scale of the rotation period. It has nothing to do with the tidal torquing of the body, which occurs over a much longer time scale.
} 
and

$$
\begin{aligned}
& v_{r}^{\mathrm{d}}=-t \frac{e^{-\psi}\left[1-8 \pi r^{2}(\mu+p)\right]}{8 \pi r^{2}(\mu+p)} k_{t r 1}^{\mathrm{d}}, \\
& v^{\mathrm{d}}=-t \frac{e^{-\psi}}{16 \pi r^{2}(\mu+p)}\left\{r^{2} f \frac{d k_{t r 1}^{\mathrm{d}}}{d r}+2\left[m-2 \pi r^{3}(\mu-p)\right] k_{t r 1}^{\mathrm{d}}\right\} .
\end{aligned}
$$

The interior solutions must be matched with the exterior solutions of Paper I at $r=R$, and this reveals that $k_{t r 1}^{\mathrm{d}}$ must satisfy the boundary condition 10

$$
k_{t r 1}^{\mathrm{d}}=0=\frac{d k_{t r 1}^{\mathrm{d}}}{d r} \quad \text { at } r=R .
$$

These conditions also follow from the requirement that $\Delta p=0$ at $r=R$, assuming that both $\mu$ and $p$ go to zero at the surface. The matching also requires $k_{t t 0}^{\mathrm{d}}$ and $k_{r r}^{\mathrm{d}}$ to agree at $r=R$ with the values listed in IV of Paper I (after taking into account the change of notation documented in footnotes 5 and 6 ). In addition, all these functions must satisfy regularity conditions at $r=0$.

We again reconsider the interior solution to allow the fluid to be static. We now set $v_{r}^{\mathrm{d}}=e^{-\psi} k_{t r}^{\mathrm{d}}$ and $v^{\mathrm{d}}=0$ to ensure that $\delta u^{r}=0=\delta u^{A}$. The conservation equations then determine $p^{\mathrm{d}}$ and imply that $k_{t r}^{\mathrm{d}}$ must be timeindependent. We next assume that the remaining metric variables are stationary, and verify that the field equations are consistent with this assumption. Our conclusion, that it is possible to construct a stationary solution to the $\ell=1$ interior problem when the fluid is static, is compatible with the computations presented in Ref. [35].

\section{Field equations: $\ell=3$}

Finally we examine the $\ell=3$ sector of the perturbation, associated with the variables $\left\{k_{t t}^{\circ}, k_{r r}^{\circ}, k^{\circ}, p^{\circ}\right\}$ and $\left\{k_{t r}^{\circ}, v_{r}^{\circ}, v^{\circ}\right\}$. The variables $v_{r}^{\circ}$ and $p^{\circ}$ are eliminated with Eqs. (3.8) and (3.9), respectively, and we find that the $t A$ and $A B$ components of the field equations allow us to further eliminate $v^{\circ}$ and $k_{r r}^{\circ}$. We are left with $k_{t t}^{\circ}$ and $k^{\circ}$ from the first group of variables, and $k_{t r}^{\circ}$ from the second group. We then assume the explicit forms $k_{t t}^{\circ}(t, r)=k_{t t 0}^{\circ}(r)$, $k^{\circ}(t, r)=k_{0}^{\circ}(r)$, and $k_{t r}^{\circ}(t, r)=k_{t r 0}^{\circ}(r)+t k_{t r 1}^{\circ}(r)$. The $t r$ component of the field equations implies that $k_{t r 0}^{\circ}$ satisfies a homogeneous differential equation, and its solution represents a $g$-mode that we allow ourselves to discard.

We are left with

$$
k_{t t}^{\circ}(t, r)=k_{t t 0}^{\circ}(r), \quad k^{\circ}(t, r)=k_{0}^{\circ}(r), \quad k_{t r}^{\circ}(t, r)=t k_{t r 1}^{\circ}(r),
$$

and the differential equations satisfied by these functions are obtained from the $t r, r r$, and $r A$ components of the field equations, respectively. We find

$$
\begin{aligned}
0= & r^{2} f \frac{d^{2} k_{t r 1}^{\circ}}{d r^{2}}+\left[3\left(m-4 \pi r^{3} \mu\right)+\left(m+4 \pi r^{3} p\right) \frac{d \mu}{d p}\right] \frac{d k_{t r 1}^{\circ}}{d r} \\
& -\frac{2}{r^{2} f}\left\{2\left[3-5 \pi r^{2}(\mu+p)+8 \pi^{2} r^{4} p^{2}\right] r^{2}-2\left[5-2 \pi r^{2}(5 \mu+7 p)\right] r m-3 m^{2}-\left(m+4 \pi r^{3} p\right)^{2} \frac{d \mu}{d p}\right\} k_{t r 1}^{\circ} \\
& -\frac{32 \pi}{3} r^{2}(\mu+p) \omega \frac{d b_{t}^{\mathrm{q}}}{d r}+\frac{16 \pi}{3}(\mu+p)\left[r^{2} \frac{d \omega}{d r}+2 \frac{3 r-7 m-4 \pi r^{3} p}{f} \omega\right] b_{t}
\end{aligned}
$$

and

$$
\begin{aligned}
0= & r \frac{d k_{t t 0}^{\circ}}{d r}+\frac{5 r+2 m-4 \pi r^{3}(\mu+p)}{m+4 \pi r^{3} p} k_{t t 0}^{\circ}-\frac{r^{3} f}{2\left(m+4 \pi r^{3} p\right)} \frac{d k_{t r 1}^{\circ}}{d r} \\
& +\frac{\left[r-4 m+2 \pi r^{3}(\mu-3 p)\right] r}{m+4 \pi r^{3} p} k_{t r 1}^{\circ}-\frac{5 e^{2 \psi}}{r\left(m+4 \pi r^{3} p\right)} k_{0}^{\circ} \\
& +\frac{r}{6}\left[\frac{\left(3-32 \pi^{2} r^{4} p^{2}\right) r^{2}-2\left(3+8 \pi r^{2} p\right) r m-2 m^{2}}{m+4 \pi r^{3} p} \frac{d \omega}{d r}-12 \omega+12\right] \frac{d b_{t}^{\mathrm{q}}}{d r}
\end{aligned}
$$

\footnotetext{
10 The calculation carried out in footnote 8 also reveals that $k_{t r 1}^{\mathrm{d}} \propto(1-r / R)^{2+n}$ when $r \rightarrow R$.
} 


$$
\begin{aligned}
& -\frac{1}{3\left(m+4 \pi r^{3} p\right)}\left\{\left[2\left(1+10 \pi r^{2} p-16 \pi^{2} r^{4} p^{2}\right) r^{2}+\left(1-16 \pi r^{2} p\right) r m-2 m^{2}\right] \frac{d \omega}{d r}\right. \\
& \left.+2\left[15 r+6 m-8 \pi r^{3}(\mu+p)\right] \omega-6\left[5 r+2 m-4 \pi r^{3}(\mu+p)\right]\right\} b_{t}^{\mathrm{q}} \\
0= & r \frac{d k_{0}^{\circ}}{d r}-\frac{5 r+2 m+8 \pi r^{3} p}{m+4 \pi r^{3} p} k_{0}^{\circ}-\frac{e^{-2 \psi} r^{5} f}{2\left(m+4 \pi r^{3} p\right)} \frac{d k_{t r 1}^{\circ}}{d r} \\
& +\frac{e^{-2 \psi} r^{3}\left[r-3 m+2 \pi r^{3}(\mu-p)\right]}{m+4 \pi r^{3} p} k_{t r 1}^{\circ}+\frac{e^{-2 \psi} r^{2}\left[5 r+2 m-4 \pi r^{3}(\mu+p)\right]}{m+4 \pi r^{3} p} k_{t t 0}^{\circ} \\
& +\frac{e^{-2 \psi} r^{4} f\left(3 r+2 m+8 \pi r^{3} p\right)}{6\left(m+4 \pi r^{3} p\right)} \frac{d \omega}{d r} \frac{d b_{t}^{\mathrm{q}}}{d r} \\
& -\frac{2 e^{-2 \psi} r^{2}}{3\left(m+4 \pi r^{3} p\right)}\left\{\left[r f\left(r+m+4 \pi r^{3} p\right)\right] \frac{d \omega}{d r}\right. \\
& \left.+\left[15 r+6 m-8 \pi r^{3}(\mu+p)\right] \omega-3\left[5 r+2 m-4 \pi r^{3}(\mu+p)\right]\right\} b_{t}^{\mathrm{q}} .
\end{aligned}
$$

The remaining variables are then given by

$$
\begin{aligned}
k_{r r}^{\circ} & =e^{-2 \psi}\left\{\frac{1}{f} k_{t t 0}^{\circ}-\frac{r^{2}}{3} \frac{d \omega}{d r} \frac{d b_{t}^{\mathrm{q}}}{d r}+\frac{2}{3}\left[r \frac{d \omega}{d r}+\frac{3(1-\omega)}{f}\right] b_{t}^{\mathrm{q}}\right\} \\
p^{\circ} & =e^{-2 \psi}\left[\frac{f}{16 \pi} \frac{d k_{t r 1}^{\circ}}{d r}+\frac{m-2 \pi r^{3}(\mu-p)}{8 \pi r^{2}} k_{t r 1}^{\circ}+\frac{1}{2}(\mu+p) k_{t t 0}^{\circ}+\frac{1}{3}(\mu+p)(3-2 \omega) b_{t}^{\mathrm{q}}\right],
\end{aligned}
$$

and

$$
\begin{aligned}
& v_{r}^{\circ}=-t \frac{e^{-\psi}\left[3-4 \pi r^{2}(\mu+p)\right]}{4 \pi r^{2}(\mu+p)} k_{t r 1}^{\circ}, \\
& v^{\circ}=-t \frac{3 e^{-\psi}}{16 \pi r^{2}(\mu+p)}\left\{r^{2} f \frac{d k_{t r 1}^{\circ}}{d r}+2\left[m-2 \pi r^{3}(\mu-p)\right] k_{t r 1}^{\circ}\right\} .
\end{aligned}
$$

As before the interior solutions must be matched with the exterior solutions of Paper I at $r=R$, and this reveals that $k_{t r 1}^{\circ}$ must satisfy the boundary condition 11

$$
k_{t r 1}^{\circ}=0=\frac{d k_{t r 1}^{\circ}}{d r} \quad \text { at } r=R
$$

These also follow from the requirement that $\Delta p=0$ at $r=R$, assuming that both $\mu$ and $p$ go to zero at the surface. The matching also requires $k_{t t 0}^{\circ}, k_{r r}^{\circ}$, and $k_{0}^{\circ}$ to agree at $r=R$ with the values listed in IV of Paper I (after taking into account the change of notation documented in footnotes 5 and 6). In addition, all these functions must satisfy regularity conditions at $r=0$.

As a final exercise we reconsider the interior solution to allow the fluid to be static. We set $v_{r}^{\circ}=e^{-\psi} k_{t r}^{\circ}$ and $v^{\circ}=0$ to ensure that $\delta u^{r}=0=\delta u^{A}$. As with $\ell=1$ we find that the conservation equations determine $p^{\circ}$ and imply that $k_{t r}^{\circ}$ is time-independent. We further assume that the remaining metric variables are stationary, and verify that the field equations are consistent with this assumption. We therefore find that a stationary solution to the $\ell=3$ interior problem is possible when the fluid is static, and this conclusion is compatible with the computations presented in Ref. [35].

\footnotetext{
11 The calculation carried out in footnote 8 also reveals that $k_{t r 1}^{\circ} \propto(1-r / R)^{2+n}$ when $r \rightarrow R$.
} 


\section{ACKNOWLEDGMENTS}

We are very grateful to John Friedman for a very helpful conversation. This work was supported by the Natural Sciences and Engineering Research Council of Canada.

[1] E. E. Flanagan and T. Hinderer, Constraining neutron star tidal Love numbers with gravitational wave detectors, Phys. Rev. D 77, 021502(R) (2008), arXiv:0709.1915.

[2] T. Hinderer, Tidal Love numbers of neutron stars, Astrophys. J. 677, 1216 (2008), erratum: Astrophys. J. 697, 964 (2009), arXiv:0711.2420.

[3] T. Damour and A. Nagar, Relativistic tidal properties of neutron stars, Phys. Rev. D 80, 084035 (2009), arXiv:0906.0096.

[4] T. Binnington and E. Poisson, Relativistic theory of tidal Love numbers, Phys. Rev. D 80, 084018 (2009), arXiv:0906.1366.

[5] P. Landry and E. Poisson, Relativistic theory of surficial Love numbers, Phys. Rev. D 89, 124011 (2014), arXiv:1404.6798.

[6] T. Hinderer, B. D. Lackey, R. N. Lang, and J. S. Read, Tidal deformability of neutron stars with realistic equations of state and their gravitational wave signatures in binary inspiral, Phys. Rev. D 81, 123016 (2010), arXiv:0911.3535.

[7] L. Baiotti, T. Damour, B. Giacomazzo, A. Nagar, and L. Rezzolla, Analytic modeling of tidal effects in the relativistic inspiral of binary neutron stars, Phys. Rev. Lett. 105, 261101 (2010), arXiv:1009.0521.

[8] L. Baiotti, T. Damour, B. Giacomazzo, A. Nagar, and L. Rezzolla, Accurate numerical simulations of inspiralling binary neutron stars and their comparison with effective-one-body analytical models, Phys. Rev. D 84, 024017 (2011), arXiv:1103.3874.

[9] J. Vines, E. E. Flanagan, and T. Hinderer, Post-1-Newtonian tidal effects in the gravitational waveform from binary inspirals, Phys. Rev. D 83, 084051 (2011), arXiv:1101.1673.

[10] F. Pannarale, L. Rezzolla, F. Ohme, and J. S. Read, Will black hole-neutron star binary inspirals tell us about the neutron star equation of state?, Phys. Rev. D 84, 104017 (2011), arXiv:1103.3526.

[11] B. D. Lackey, K. Kyutoku, M. Shibata, P. R. Brady, and J. L. Friedman, Extracting equation of state parameters from black hole-neutron star mergers. I. Nonspinning black holes, Phys. Rev. D 85, 044061 (2012), arXiv:1109.3402.

[12] T. Damour, A. Nagar, and L. Villain, Measurability of the tidal polarizability of neutron stars in late-inspiral gravitationalwave signals, Phys. Rev. D 85, 123007 (2012), arXiv:1203.4352.

[13] J. S. Read, L. Baiotti, J. D. E. Creighton, J. L. Friedman, B. Giacomazzo, K. Kyutoku, C. Markakis, L. Rezzolla, M. Shibata, and K. Taniguchi, Matter effects on binary neutron star waveforms, Phys. Rev. D 88, 044042 (2013), arXiv:1306.4065.

[14] J. E. Vines and E. E. Flanagan, First-post-Newtonian quadrupole tidal interactions in binary systems, Phys. Rev. D 88, 024046 (2013), arXiv:1009.4919.

[15] A. Maselli, L. Gualtieri, and V. Ferrari, Constraining the equation of state of nuclear matter with gravitational wave observations: Tidal deformability and tidal disruption, Phys. Rev. D 88, 104040 (2013), arXiv:1310.5381.

[16] B. D. Lackey, K. Kyutoku, M. Shibata, P. R. Brady, and J. L. Friedman, Extracting equation of state parameters from black hole-neutron star mergers: Aligned-spin black holes and a preliminary waveform model, Phys. Rev. D 89, 043009 (2014), arXiv:1303.6298.

[17] M. Favata, Systematic parameter errors in inspiraling neutron star binaries, Phys. Rev. Lett. 112, 101101 (2014), arXiv:1310.8288.

[18] K. Yagi and N. Yunes, Love number can be hard to measure, Phys. Rev. D 89, 021303 (2014), arXiv:1310.8358.

[19] T. Delsate, I-Love irrotationally (2015), arXiv:1504.07335.

[20] K. Yagi and N. Yunes, I-Love-Q, Science 341, 365 (2013), arXiv:1302.4499.

[21] K. Yagi and N. Yunes, I-Love-Q relations in neutron stars and their applications to astrophysics, gravitational waves, and fundamental physics, Phys. Rev. D 88, 023009 (2013), arXiv:1303.1528.

[22] D. D. Doneva, S. S. Yazadjiev, N. Stergioulas, and K. D. Kokkotas, Breakdown of I-Love-Q universality in rapidly rotating relativistic stars, Astrophys. J. 781, L6 (2014), arXiv:1310.7436.

[23] A. Maselli, V. Cardoso, V. Ferrari, L. Gualtieri, and P. Pani, Equation-of-state-independent relations in neutron stars, Phys. Rev. D 88, 023007 (2013), arXiv:1304.2052.

[24] K. Yagi, Multipole Love relations, Phys. Rev. D 89, 043011 (2014), arXiv:1311.0872.

[25] B. Haskell, R. Ciolfi, F. Pannarale, and L. Rezzolla, On the universality of I-Love-Q relations in magnetized neutron stars, Mon. Not. R. Astron. Soc. 438(1), L71 (2014), arXiv:1309.3885.

[26] S. Chakrabarti, T. Delsate, N. Gürlebeck, and J. Steinhoff, I-Q relation for rapidly rotating neutron stars, Phys. Rev. Lett. 112, 201102 (2014), arXiv:1311.6509.

[27] D. Bini, T. Damour, and G. Faye, Effective action approach to higher-order relativistic tidal interactions in binary systems and their effective one body description, Phys. Rev. D 85, 124034 (2012), arXiv:1202.3565.

[28] S. Chakrabarti, T. Delsate, and J. Steinhoff, New perspectives on neutron star and black hole spectroscopy and dynamic tides (2013), arXiv:1304.2228.

[29] S. Chakrabarti, T. Delsate, and J. Steinhoff, Effective action and linear response of compact objects in Newtonian gravity, Phys. Rev. D 88, 084038 (2013), arXiv:1306.5820.

[30] S. R. Dolan, P. Nolan, A. C. Ottewill, N. Warburton, and B. Wardell, Tidal invariants for compact binaries on quasicircular orbits, Phys. Rev. D 91, 023009 (2015), arXiv:1406.4890. 
[31] D. Bini and T. Damour, Gravitational self-force corrections to two-body tidal interactions and the effective one-body formalism, Phys. Rev. D 90, 124037 (2014), arXiv:1409.6933.

[32] E. Poisson, Tidal deformation of a slowly rotating black hole, Phys. Rev. D 91, 044004 (2015), arXiv:1411.4711.

[33] P. Landry and E. Poisson, Tidal deformation of a slowly rotating material body: External metric, Phys. Rev. D 91, 104018 (2015), arXiv:1503.07366.

[34] P. Pani, L. Gualtieri, A. Maselli, and V. Ferrari, Tidal deformations of a spinning compact object, Phys. Rev. D 92, 024010 (2015), arXiv:1503.07365.

[35] P. Pani, L. Gualtieri, and V. Ferrari, Tidal Love numbers of a slowly-spinning neutron star (2015), arXiv:1509.02171.

[36] K. H. Lockitch, N. Andersson, and J. L. Friedman, Rotational modes of relativistic stars: Analytic results, Phys. Rev. D 63, 024019 (2000), arXiv:gr-qc/0008019.

[37] K. H. Lockitch, J. L. Friedman, and N. Andersson, Rotational modes of relativistic stars: Numerical results, Phys. Rev. D 68, 124010 (2003), arXiv:gr-qc/0210102.

[38] N. Andersson, A new class of unstable modes of rotating relativistic rtars, Astrophys. J. 502, 708 (1998), arXiv:grqc/9706075.

[39] S. Chandrasekhar, Solutions of two problems in the theory of gravitational radiation, Phys. Rev. Lett. 24, 611 (1970).

[40] J. L. Friedman and B. F. Schutz, Secular instability of rotating Newtonian stars, Astrophys. J. 222, 281 (1978).

[41] P. Landry and E. Poisson, Gravitomagnetic response of an irrotational body to an applied tidal field, Phys. Rev. D 91, 104026 (2015), arXiv:1504.06606.

[42] A. Campolattaro and K. S. Thorne, Non-radial pulsation of general relativistic stellar models. V. Analytical analysis for $l=1$, Astrophys. J. 159, 847 (1970).

[43] J. L. Friedman and N. Stergioulas, Rotating relativistic stars (Cambridge University Press, Cambridge, 2013).

[44] M. Favata, Are neutron stars crushed? Gravitomagnetic tidal fields as a mechanism for binary-induced collapse, Phys. Rev. D 73, 104005 (2006), arXiv:astro-ph/0510668.

[45] K. S. Thorne, Non-radial pulsation of general relativistic stellar models. III. Analytic and numerical results for neutron stars, Astrophys. J. 158, 1 (1969). 